\title{
Barthes et l'« hésitation " proustienne ou le cheminement des deux côtés de La Chambre claire
}

Guillaume Bellon

\section{CpenEdition}

\section{Journals}

Édition électronique

URL : http://journals.openedition.org/genesis/1166

DOI : 10.4000/genesis. 1166

ISSN : 2268-1590

Éditeur :

Presses universitaires de Paris Sorbonne (PUPS), Société internationale de génétique artistique littéraire et scientifique (SIGALES)

Édition imprimée

Date de publication : 15 juin 2013

Pagination : 165-177

ISBN : 978-2-84050-893-9

ISSN : 1167-5101

\section{Référence électronique}

Guillaume Bellon, «Barthes et l'« hésitation » proustienne ou le cheminement des deux côtés de La Chambre claire », Genesis [En ligne], 36 | 2013, mis en ligne le 16 juin 2015, consulté le 19 avril 2019. URL : http://journals.openedition.org/genesis/1166 ; DOI : 10.4000/genesis.1166 


\title{
Barthes et l'« hésitation " proustienne ou le cheminement des deux côtés de La Chambre claire
}

\author{
Guillaume Bellon
}

$\mathrm{O}$ n connaît le vertige des listes et leur pouvoir d'illimitation 1 : listes de «choses à faire » ou « à ne pas oublier », elles ouvrent au-delà du sujet qui les établit le temps d'un possible serein, où tout ce qui nous pèse trouverait à s'ordonner. Les sujets que nous sommes s'adonnent moins (et faut-il le regretter?) au geste inverse, celui du tableau récapitulatif périodisant les étapes d'une vie, ses articulations décisives comme ses bifurcations. À cet étrange exercice, Barthes s'essaie dans le tableau «Phases » du Roland Barthes par Roland Barthes ${ }^{2}$ (voir fig. 1). Parmi les influences qu'il cite alors (Gide, Nietzsche, mais aussi Kristeva ou Sollers), on chercherait en vain le nom de Proust. En 1975 - date de parution de l'ouvrage dans la collection «Écrivains de toujours » au Seuil - l'essayiste a pourtant consacré plusieurs études à l'auteur de la Recherche, dont l'article "Proust et les noms », repris en 1964 dans les Essais critiques $^{3}$; dans les années qui suivront, " Ça prend » (réflexion publiée en 1979 sur le départ d'écriture de la somme proustienne), comme un an auparavant la conférence «Longtemps, je me suis couché de bonne heure... » attesteront de la présence vive de Proust dans l'imaginaire barthésien ${ }^{4}$. Faudrait-il conclure alors à une assomption progressive de l'influence proustienne, un temps passée sous silence et, notamment à l'occasion de la première année du cours La Préparation du roman (en 1978) ${ }^{5}$, in fine revendiquée?

Il faut se méfier des influences, et la page à laquelle nous faisons référence dans le Roland Barthes ne dit pas autre chose : « le découpage d'un temps, d'une œuvre, en phases d'évolution » relève d'une " opération imaginaire 6 ». Si par là, « on se fait intelligible », il s'agit bien d'une démarche conduite par l'auteur lui-même en vue d'orienter la saisie de son œuvre - c'est tout le problème du Roland Barthes. Plus encore, le tableau dressé par
Barthes s'organise en trois colonnes : à l' «intertexte » («musique de figures, de métaphores, de pensées-mots» précise-t-il) correspond un « genre » (la mythologie, la sémiologie ou encore la "moralité », définie comme "pensée du corps en état de langage ») et autant d'« œuvres » : ainsi à la case « Nietzsche » correspond le désir d'écrire les «moralités » que seraient - troisième case - Le Plaisir du texte ou, donc, l'essai de 1975 que nous citons. Et si Proust ne trouvait pas à s'inscrire là parce que son influence était d'un autre ordre ? S'il n'apparaissait pas précisément en ce qu'il relève du « hors case » ? C'est ce qu'affirme Barthes, lors de la conférence « Longtemps, je me suis couché de bonne heure...», prononcée le 19 octobre 1978 au Collège de France, et quelques semaines plus tard (le 2 décembre) à l'ouverture du cours La Préparation $d u$ roman. Au sein d'une même institution, le Collège de France, et à deux reprises, une pensée identique est développée autour de la figure proustienne.

Ce moment dans la pensée barthésienne, moment d'appel à Proust, on peut en rappeler le caractère solennel : Barthes, revenant sur ses travaux passés, fait état d'une grande lassitude, d'un véritable ennui. Au moyen d'un

1. C'est à U. Eco que j'emprunte l'expression : voir Vertige de la liste, Paris, Flammarion, 2009.

2. Le tableau se trouve reproduit dans le Roland Barthes par Roland Barthes, p. 718 du tome IV des Euvres complètes, éditées par É. Marty (Paris, Éditions du Seuil, 2002). Celles-ci seront dorénavant abrégées en $O C$, mention suivie du numéro du volume auquel se reporter.

3. R. Barthes, «Proust et les noms », dans $O C$, t. II, p. 66-77.

4. R. Barthes, « Ça prend» $(O C$, t. V, p. 654-656) et «Longtemps, je me suis couché de bonne heure » $(O C, \mathrm{t}$. V, p. 459-470).

5. R. Barthes, La Préparation du roman, éd. N. Léger, Paris, Éditions du Seuil, 2003.

6. R. Barthes, Roland Barthes, op. cit., p. 719. 


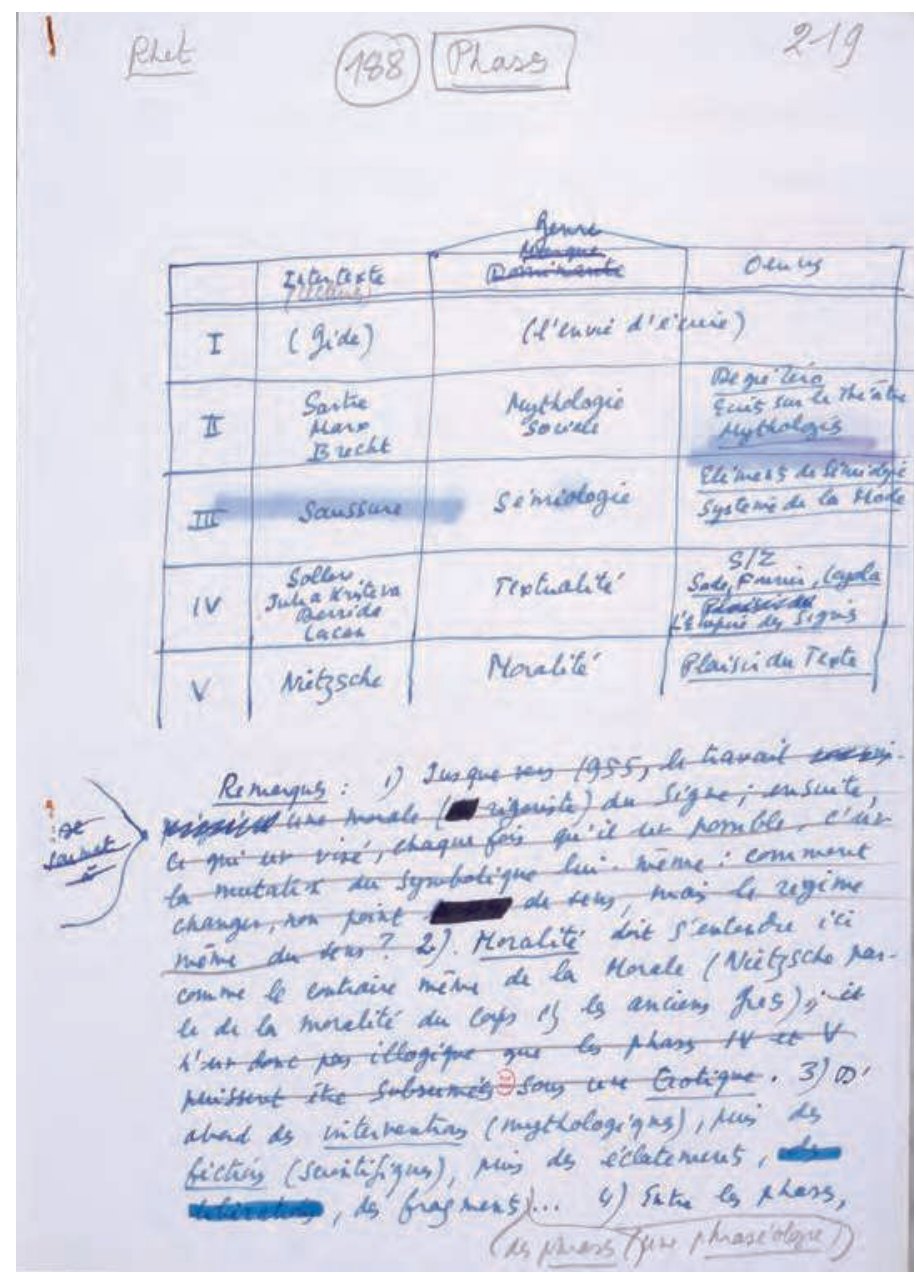

Fig. 1 : Roland Barthes par Roland Barthes

(BnF, NAF 28630, Roland Barthes par Roland Barthes, manuscrit, dossier 3, fo 219)

terme emprunté à la théologie médiévale, il revient sur ce sentiment qui constituait déjà la première figure du cours Comment vivre ensemble : l'Acédie 7 . Cette inappétence, désignant originairement le désinvestissement du moine, équivaut, pour l'auteur, à prendre conscience du fait qu' " il n'y a plus de hors case ». Et de s'indigner : «Quoi ? Toujours, jusqu'à ma mort, je vais écrire des articles, faire des cours, des conférences - ou mieux des livres - sur des sujets qui seuls varieront (si peu !) 8 ? » C'est sur ce fond de « vague à l'âme », de « tristesse » ou encore de « découragement ${ }^{9} »$ que s'inscrit la sollicitation de Proust, son évocation autant que son invocation. Si la teneur de cette confiance placée en un auteur, développée sans grande variation entre la conférence et le cours, est connue (et s'il

7. Voir R. Barthes, Comment vivre ensemble. Simulations de quelques espaces romanesques, éd. Cl. Coste, Paris, Éditions du Seuil/IMEC, 2002, p. 53 : «Sentiment, état du moine qui désinvestit de l'ascèse, qui n'arrive plus à investir en elle ( $\neq$ qui perd la foi). Ce n'est pas une perte de croyance, c'est une perte d'investissement ».

8. R. Barthes, La Préparation du roman, op. cit., p. 27. La conférence formulait la révolte en ces termes : «parce que la case est dessinée, parce qu'il n'y a plus de "hors case", le travail que je vais y loger prend une sorte de solennité » («Longtemps... », art. cité, p. 466).

9. R. Barthes, Comment vivre ensemble, op. cit., p. 53. 
suffit de se reporter aux textes publiés pour la retrouver ${ }^{10}$ ), les manuscrits barthésiens permettent cependant d'en mesurer toute l'intensité : c'est à cette première lecture que nous voudrions travailler.

\section{Avancer « sous le masque de Proust »}

À la façon de Barthes assumant, dans le Comment vivre ensemble, d' " ouvrir des dossiers », c'est-à-dire avec une précaution identique, ouvrons donc le dossier génétique de la conférence consacrée à Proust. Trois manuscrits, dont le détail est instructif, sont conservés à la Bibliothèque nationale : deux manuscrits (complets et entièrement rédigés) relatifs à la conférence d'octobre 1978, et un troisième, qui n'est qu'une copie sans grande modification du texte définitif prononcé au Collège, et adapté en vue de la prestation de Barthes à New York, le mois suivant ${ }^{11}$. Si ce troisième manuscrit n'offre ici qu'un intérêt restreint, c'est le passage du premier au deuxième qui retiendra toute notre attention. La première version manuscrite du texte ne renseigne pas seulement sur la dispositio d'abord envisagée par Barthes ${ }^{12}$; elle donne également à lire une expression moins contournée, plus directement affective, de l'identification à Proust.

Dès le premier manuscrit de la conférence, Barthes inscrit l'annonce qu'il lira devant les auditeurs du Collège : «Ce sera, si vous voulez bien : Proust et moi (quelle prétention ! J'y reviendrai) 13 »; mais là où la suite du propos, dans la version définitive, s'appuie sur l'exemple nietzschéen («Nietzsche ironisait sur l'usage que les Allemands faisaient de la conjonction "et" : "Schopenhauer et Hartmann", raillait-il14 »), le feuillet manuscrit met en jeu une formulation plus directe : " moi derrière Proust, m'avançant sous le masque de Proust » (fo 1, voir fig. 2). L'image inédite du «masque de Proust» ne doit pas être sous-estimée ; elle entre en résonance avec cet aveu du Roland Barthes (ce petit bréviaire, mais miné parce qu'exposé par lui-même, de l'imaginaire barthésien) : le critique reconnaissait que dans son travail, l'important «n'est cependant pas l'auteur dont je parle, mais plutôt ce qu'il $m$ 'amène à dire de lui : je m'influence moi-même avec sa permission : ce que je dis de lui m'oblige à le penser de moi (ou à ne pas le penser), etc. »15. Il s'agit moins cependant d'une réelle confusion (de postures, d'identités) que d'une fusion d'abord affective : Barthes revendique vouloir se «mêl[er] "amoureusement" (Nietzsche) à cette œuvre monumentale, ou du moins à son Projet » (fo 1). Pareil lexique amoureux, le conférencier « qui parle et qui dit ${ }^{16}$ » préférera la recouvrir d'un voile de pudeur.

Or, c'est bien d'une rencontre amoureuse que fait état cette première version - ou plutôt, selon la très belle expression qu' on lit au feuillet 6 , d'une de ces « rencontres d'identité » dont le rayonnement ultérieur définit la vie de la lecture. Barthes rappelle par là que s'identifier à quelqu'un, c'est d'abord reconnaître en lui un possible autre soi-même (qu'il faille, ou non, pour parvenir à cette ressemblance, cette identité, faire l'épreuve d'une modification de soi). C'est également déceler en cet autre choisi une même difficulté, l'espace d'un souci commun :

[...] le point actif de l'identification est celui-ci : Proust s'est posé le problème de l'œuvre à faire, de l'œuvre-devant-soi : qu'on a devant soi, qu'on veut, mais qu'on est impuissant à faire « prendre » : c'est d'ailleurs tout son roman : l'œuvre au futur (l'œuvre se trouve écrite alors qu'à la dernière page on trouve le moyen de l'écrire : geste (au masc. et au féminin) de tous ceux qui veulent écrire - et je dirai presque : geste épique, mythique, ce par quoi nous nous retrouvons d'une façon si brûlante, si concernée en lui (fo 6 , voir fig. 3).

10. On consultera notamment 1'article de K. Yacavone, « Barthes et Proust : la Recherche comme aventure photographique », dans «L'écrivain préféré », Fabula LHT (Littérature, histoire, théorie), n 4, mars 2008, URL : <www.fabula.org/lht/4/Yacavone.html> (page consultée le 26 novembre 2012).

11. R. Barthes, BnF, trois ensembles : 23 f. mss, 19 f. mss et 20 f. tapuscrits avec corrections manuscrites.

12. Notamment quant à l'insertion de l'exemple de Dante, envisagée dès le premier feuillet et reportée dans la version définitive en deuxième partie de la conférence. Sur ce point, je renvoie à mon livre : Une parole inquiète. Barthes et Foucault au Collège de France, Grenoble, ELLUG, 2012, en particulier p. 87-93.

13. R. Barthes, NAF 28630, « Longtemps je me suis couché de bonne heure » première version manuscrite, fo 1 . Les références à ce manuscrit se feront pour la suite de ce développement dans le corps du texte, entre parenthèses.

14. R. Barthes, « Longtemps... », art. cité, p. 459.

15. R. Barthes, «Qu'est-ce que 1'influence? », dans Roland Barthes, op. cit., p. 683.

16. Pour reprendre ici l'ouverture bien connue des Fragments d'un discours amoureux (OC, t. V, p. 35) : «C'est donc un amoureux qui parle et qui dit...». 


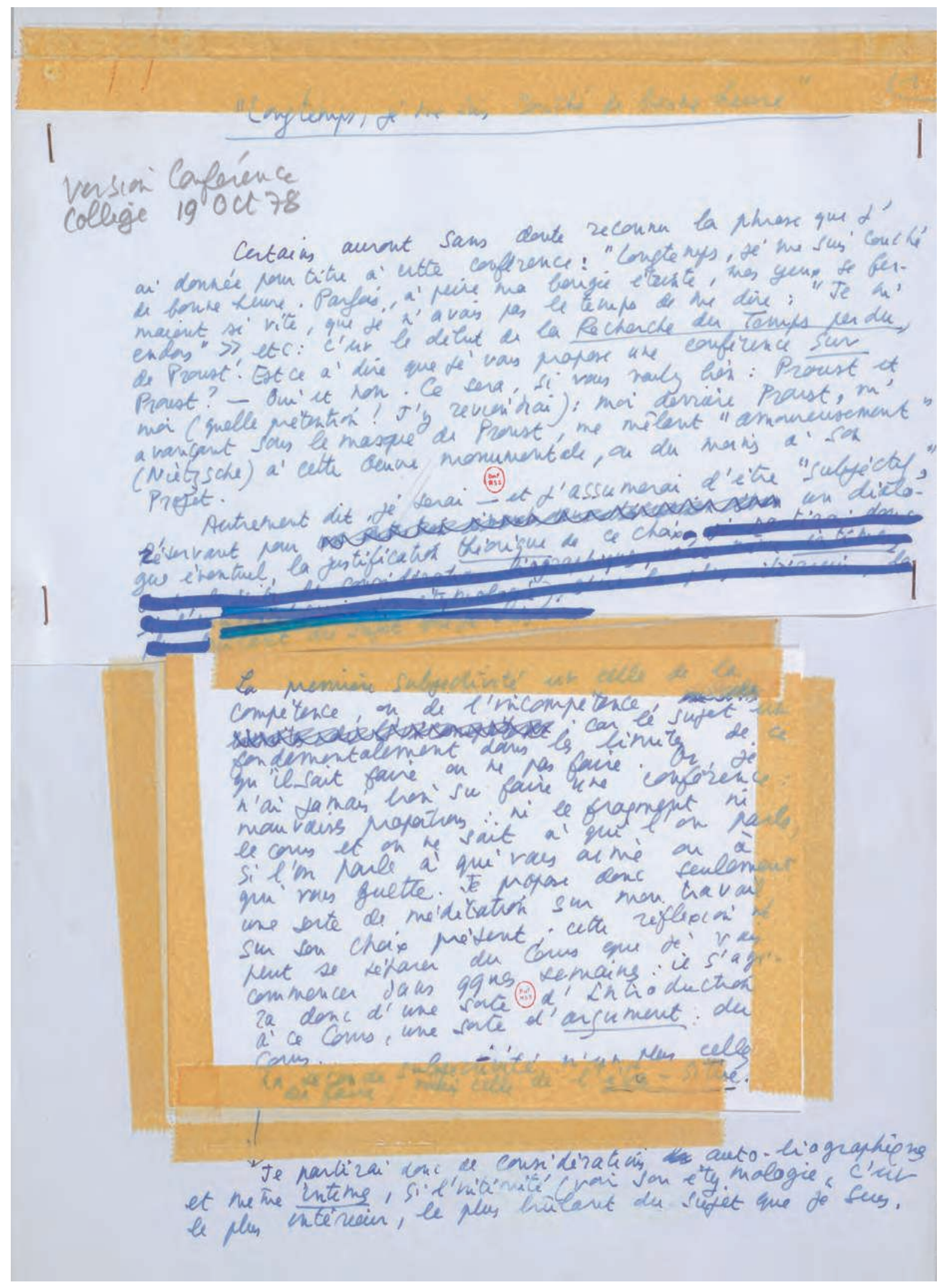

Fig. 2 : «Longtemps, je me suis couché de bonne heure », première version manuscrite de la conférence (BnF, NAF 28630, fo 1) 


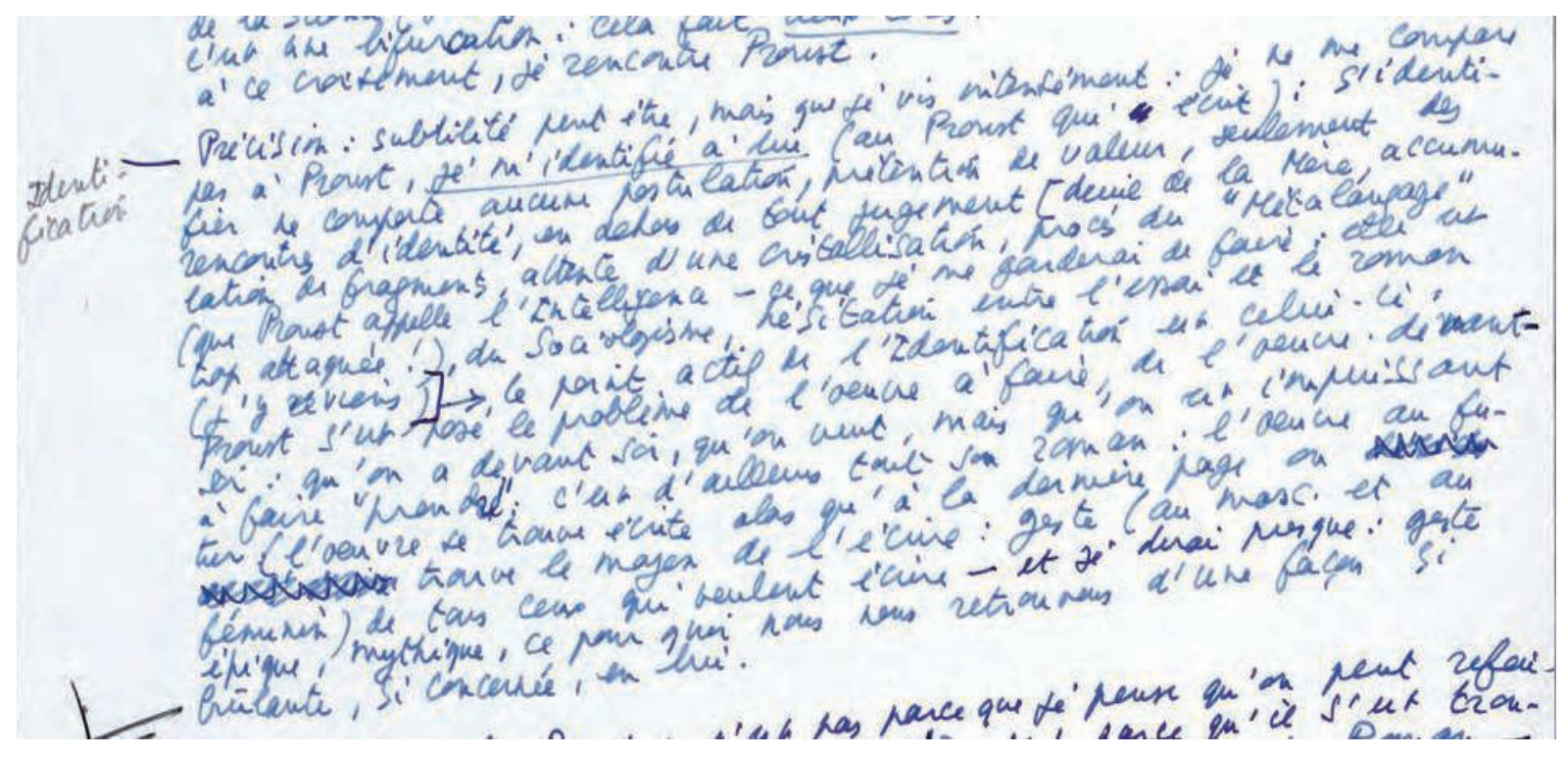

Fig. 3 : «Longtemps, je me suis couché de bonne heure », première version manuscrite de la conférence (BnF, NAF 28630, fo 6, détail)

Proust est alors promu (en un regard croisé du côté de Dante) «Virgile » dans cette épopée, cette quête - selon une comparaison que ne consacre pas la conférence, mais la première séance du cours ${ }^{17}$. Reprenons le fil de la conférence : s'il est un guide, c'est en ce qu'il offre au sujet Barthes désirant sortir de l'essai « un modèle libératoire, l'initiation d'une liberté d'écrire » (fo 7).

L'auteur de la Recherche désignerait ainsi une possible conduite de son existence (et de l'existence de soi dans l'écriture) que Barthes repère pour mieux la répéter dans sa propre vie. Pour le dire au moyen des termes mis en jeu par Marielle Macé dans son récent essai, Barthes cherche, dans sa «façon de lire » Proust, une «manière d'être ", et d'être dans l'écriture18. Dès lors, Proust ne figure pas véritablement une « influence »; il faudrait pour cela pouvoir encore penser sa vie en termes de tableau, de cases multiples, et cela n'est plus possible («ce qu'on a fait, travaillé, écrit, apparaît comme voué à la répétition 19 »). Ce qu'il semble offrir à Barthes, c'est un modèle élu, une conduite choisie dans la gamme des conduites passées. Comme Rimbaud, lors de la seconde année de La Préparation du roman, est le contre-modèle du « sabordage spectaculaire de l'écrire », de l'a-graphie 20 , Proust sera la figure tutélaire d'un désir d'écriture encore en quête, pour Barthes, de sa juste forme d'expression. En glissant ses pas dans les pas de l'auteur de la Recherche, il s'agirait ainsi d'aller au-devant de « la découverte d'une nouvelle pratique d'écriture $21 »-\langle$ nouvelle pratique » qui serait moins le « sabordage » que la subversion de la posture essayistique précédemment adoptée. C'est autour du désir d'une « tierce forme », entre le Roman et l'Essai22, repérée chez Proust, que se cristallisent cette découverte et la possible subversion qu'elle semble promettre.

17. Voir R. Barthes, La Préparation du roman, op. cit., p. 26. 18. L'essai de M. Macé, Façons de lire, manières d'être (Paris, Gallimard, 2011), réfléchit aux modalités d'insertion de la lecture dans la vie et à l'insistance de certaines figures littéraires et des possibles qu'elles ouvrent dans le champ d'une existence qualifiée (i.e. en quête d'une certaine qualité - ici exemplairement d'écriture).

19. R. Barthes, «Longtemps... », art. cité, p. 466.

20. R. Barthes, La Préparation du roman, op. cit., p. 210.

21. Ibid., p. 29.

22. Voir R. Barthes, «Longtemps... », art. cité, p. 460-461. 
Où chercher les traces de cette liberté ? On ne trouve, dans le fonds Roland Barthes, ni carnet de notes, ni esquisse ou brouillon qui témoigneraient d'une première textualisation exposant les aléas, doutes ou revirements d'un scripteur en lutte avec l'écriture par lui jusque-là pratiquée : il faut donc convenir d'une mutation indécelable à même les brouillons - mais non moins certaine à lire les derniers textes de l'auteur, notamment La Chambre claire, en 198023. Déformation de notre lecture (parce qu'il s'agit là du dernier ouvrage publié par Barthes), ou reconnaissance d'une évidence travaillant l'ouvrage : La Chambre claire met en œuvre la liberté d'une distance marquée au genre de l'essai - à son côté24. Si c'est là, sans doute, un lieu commun dans la réception actuelle de cette Note sur la photographie (selon son sous-titre), on peut néanmoins vouloir comparer celle-ci aux «quelques réflexions » livrées quant à la photographie, dans le cadre de La Préparation $d u$ roman $^{25}$. C'est à cet égard qu'il semble pertinent, autour du rôle libérateur joué par la figure proustienne dans l'écriture barthésienne, d'étudier plusieurs dossiers génétiques : celui de la conférence "Longtemps... », mais aussi du livre de 1980 ainsi que les archives (manuscrites et sonores) relatives à la deuxième année (nous avons jusqu'à présent cité seulement la première) du cours La Préparation du roman ${ }^{26}$. Ces dossiers et documents, les liens les unissant peuvent paraître parfois ténus, et plus encore, on ne les soumettra peut-être pas au jeu serré des études génétiques; il semble néanmoins qu'à les tenir ensemble, on mesure ce cheminement dans la volition barthésienne d'une nouvelle écriture (posée dans la conférence ou le cours), sa mise à l'épreuve et son inscription subtile dans le livre. Ces relations complexes, autotextuelles, d'un document à l'autre, on pourrait en rendre compte au moyen d'une image botanique choisie par Barthes lui-même. Il s'agit en effet de rendre sensible, d'un document à l'autre, d'une amorce de réflexion ou d'écriture à l'autre, ce réseau d'insistances, ce retour d'interrogation quant à la forme à choisir, en un mot, ce «marcottage »- et l'on retrouve le terme par lequel Barthes rend compte, dans « Ça prend », de l'écriture de la Recherche : ce qui, «plant[é] ici, souvent discrètement [...], se retrouve bien plus tard, par enjambement au-dessus d'une infinité d'autres rela- tions 27 ». Ainsi - c'est l'hypothèse que cet article voudrait explorer - de 1' "hésitation proustienne », «plantée » lors de la conférence ou du cours, et qui «se retrouve » dans La Chambre claire.

\section{Le côté de l'Essai}

Retraçons d'abord brièvement les parentés entre le cours et le livre28. Soucieuse de définir la « lisibilité » du haïku, son «effet de réel », la séance du 15 février 1979 de

23. R. Barthes, La Chambre claire, dans $O C$, t. V. Les références à ce texte (abrégé $C C$ ) seront désormais données dans le corps du texte, entre parenthèses. Le dossier génétique de ce livre a fait l'objet d'une présentation et d'une étude scrupuleuses : voir J.-L. Lebrave, « Point sur la genèse de La Chambre claire », dans Genesis, $\mathrm{n}^{\circ}$ 19, « Roland Barthes », 2002, p. 79-108.

24. Il est une autre possibilité, aujourd'hui bien mise au jour et sur laquelle je ne reviens pas : celle de faire des quelques feuillets manuscrits présentés dans les Euvres complètes sous le titre « Vita Nova » le lieu d'expérimentation d'une sortie de l'Essai - et d'un essayage au roman. Voir, notamment, l'analyse proposée par Cl. Amigo Pino dans son article « Le roman du temps perdu. Le mythe de Proust et la recherche de Barthes » (dans Recherches et travaux, « Le devenir-roman des Mythologies de Barthes », $\mathrm{n}^{\circ} 77$, dir. G. Bellon et P. Vachaud, Grenoble, ELLUG, 2010, p. 46-56).

25. C'est le sens de la note de Nathalie Léger : "Ce passage consacré à la photographie constitue l'esquisse de ce que Roland Barthes développera quelques mois plus tard dans La Chambre claire. Note sur la photographie, rédigé après le cours, entre le 15 avril et le 3 juin 1979, particulièrement pour ce qui relève de la tentative d'identification du "noème de la photographie" ", dans R. Barthes, La Préparation du roman, op. cit., n. 2, p. 113.

26. Le cours sera abrégé : $P R$ (références données entre parenthèses). Pour la transcription des archives sonores (entre soufflets simples), il s'agit de la séance du 15 février 1979, disponible sur le CD publié par le Seuil.

27. Je reproduis la citation complète : « [Q]uand on sait l'importance des retours, coïncidences, renversements, tout au long de la Recherche, et combien Proust était fier de cette composition par enjambements, qui fait que tel détail insignifiant, donné au début du roman, se retrouve à la fin, comme poussé, germé, épanoui, on peut penser que ce que Proust a découvert, c'est l'efficacité romanesque de ce que l'on pourrait appeler le "marcottage" des figures : plantée ici, souvent discrètement (disons, au hasard, par exemple : la dame en rose), une figure se retrouve bien plus tard, par enjambement au-dessus d'une infinité d'autres relations, fonder une nouvelle souche (Odette) » (R. Barthes, « Ça prend », art. cité, p. 656).

28. Les pages qui suivent reprennent certains exemples abordés dans Une parole inquiète (op. cit., p. 116-125), en orientant l'analyse autour de la «tierce forme » proustienne. 
La Préparation du roman présente un ensemble de notes sur la « photo » qu'à l'oral, le professeur introduit comme $<$ petite digression>. «Lisibilité », « effet de réel » : ces termes, véritables mots-valeurs dans la pensée barthésienne, sont au cœur de La Chambre claire ; on s'étonnera néanmoins de les trouver d'abord au départ d'une étude portant sur le haïku. Barthes s'en montre conscient et se justifie par le mot de Proust - tiens donc... - dans le Contre Sainte-Beuve : il s'agit d' " entrer dans un art par un autre » $(P R$, p. 113). Quitte à abandonner le premier dès lors qu'apparaît maîtrisé le second : le livre, dans sa version publiée, présente une seule référence à la forme poétique qui aura occupé le professeur durant un an au Collège de France, et qui semble dès lors bien absente de son dernier ouvrage. Mentionnons-la : «Ceci rapproche la Photographie du Haïku. Car la notation d'un haïku, elle aussi, est indéveloppable : tout est donné, sans provoquer l'envie ou même la possibilité d'une expansion rhétorique » $(C C, \mathrm{p} .828)$. « [V]ous ne pouvez rien ajouter à une photo », avait déjà avancé le cours ( $P R$, p. 117), pointant l'impossibilité de la photo à susciter le mouvement du rêve, sur laquelle s'achève également le développement du livre : «ni le Haïku, ni la Photo ne font "rêver" » $(C C$, p. 828). Les positions s'échangent ; c'est à chaque fois comme terme d'une complémentarité qu'au sein du cours sur le haïku, la photo est convoquée, quand, au cœur du livre sur la photo, le haïku fait un retour furtif dans le propos de Barthes. Inutile, on en conviendra, de faire des notes de cours l'avant-texte, même lointain, de cette Note sur la photographie.

La lecture des notes de cours joue à un autre niveau ; leur prise en compte comme élément « flottant» dans la genèse du texte, comme matérialisation à un moment donné d'une réflexion en chemin, permet ainsi d'expliquer, peut-être, la présence, parmi les différents documents préparatoires réunis par Barthes, de deux fiches sur Benveniste29. Leur réunion, parmi les nombreux documents préparatoires colligés par Barthes, indique sans doute l'une des voies explorées, puis abandonnées, lors de la rédaction du livre. Le texte définitif, publié en 1980, ne porte aucune mention des théories du linguiste 30 quand la séance du 15 février s'appuie explicitement sur elles. Devant les auditeurs du Collège de France, Barthes, après avoir comparé haïku et photo autour du détail, poursuit : "Le "Ça a été" de la Photo et du haïku peut être interrogé sous une autre pertinence : celle de la catégorie temporelle dont ils relèvent » $(P R, \mathrm{p} .117-118)$. Le développement du cours n'est alors pas sans rapport avec les quelques références portées sur les deux fiches de lecture de Benveniste :

Où se place le «Ça a été » du haïku ? Bien que très souvent écrit au présent [...], on écrit (dans les traductions) sans verbe [...]; évident que cela renvoie au passé : non pas l'aoriste (ce fut), mais bien entendu le parfait, temps de l'évocation, du lien affectif entre ce qui a eu lieu et ce que je suis en me remémorant [...] (ibid., p. 118).

Pédagogie oblige, Barthes rappelle ensuite le contraste mis au jour par Benveniste entre les deux régimes, à l'appui des mêmes références que celles portées sur les deux fiches retrouvées dans le dossier préparatoire de La Chambre claire: Problèmes de linguistique générale, I, p. 239-240. Si le parfait représente $<$ le temps du discours $>$, $<$ l'aoriste sert à pointer l'événement hors de la personne d'un narrateur> : <c'est la forme typique de l'histoire, du récit historique $>$, explicite-t-il encore, $<$ forme qui a disparu, vous le savez, du discours parlé>. L'aoriste serait ainsi l'équivalent, dans le système des temps verbaux, du noème spécifique du haïku. «Et la Photo ? Je ne sais ; à analyser plus tard », lance le professeur, avant de rendre compte rapidement de son hypothèse de travail : «Certainement : très largement au parfait - mais peut-être des photos à l'aoriste (par exemple, vignettes du Larousse) » (loc. cit.). L'intuition ici exposée, nul approfondissement n'en est proposé dans La Chambre claire, n'était cette courte parenthèse : « la Photo n'est jamais, en essence, un souvenir (dont l'expression grammaticale serait le parfait, alors que le temps de la Photo, c'est plutôt l'aoriste) » $(C C$, p. 863). Pareille mention parembolique, pour discrète qu'elle se présente, synthétise le développement contrastif entre aoriste et

29. Il s'agit des fiches numérotées [287] et [288] dans le fonds. Voir leur transcription dans J.-L. Lebrave, « Point sur la genèse de La Chambre claire », art. cité, p. 82.

30. Pour J.-L. Lebrave, « la relecture et l'intégration des fiches Benveniste, loin d'avoir une finalité réflexive, répondait à une raison pratique, liée à la genèse même du texte : dans le travail de mise en place du registre narratif propre à La Chambre claire, les analyses de Benveniste ont peutêtre aidé Barthes à résoudre un problème d'écriture » (ibid., p. 85). 


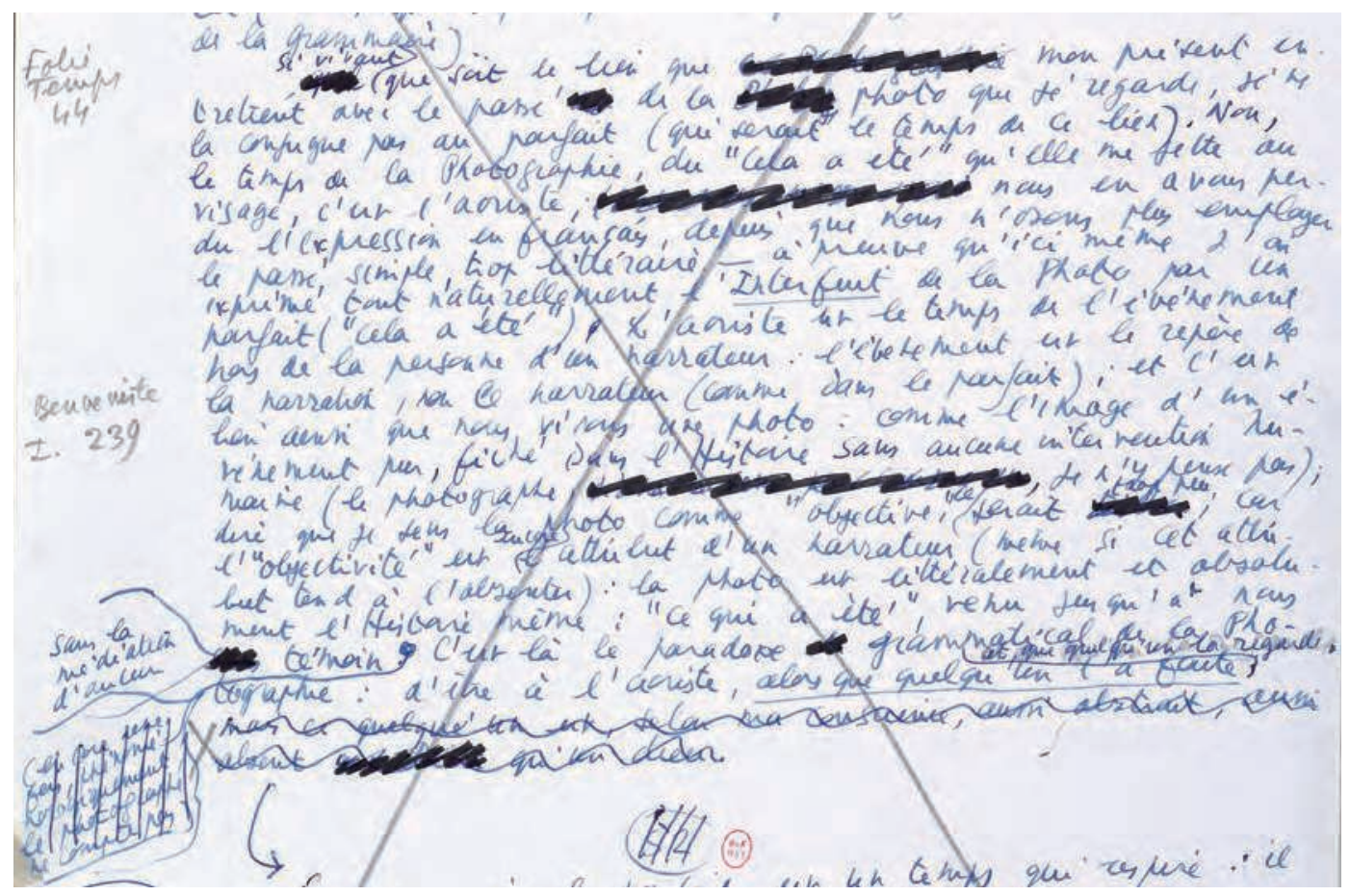

Fig. 4 : Manuscrit de La Chambre claire, fo 40, détail (BnF, NAF 28630)

parfait, longuement détaillé par le professeur dans le cadre de La Préparation du roman, et que l'on retrouvera en particulier dans une version antérieure du livre. On peut lire ainsi, au bas du feuillet numéroté 40 dans le manuscrit du livre, ce développement biffé (voir fig. 4) :

Si vivant que soit le lien que mon présent entretient avec le passé de la Photo que je regarde, je ne le conjugue pas au parfait (qui serait le temps de ce lien). Non, le temps de la Photographie, du «cela a été » qu'elle me jette au visage, c'est l'aoriste ; nous en avons perdu l'expression en français depuis que nous n'osons plus employer le passé simple, trop littéraire - à preuve qu'ici même, j'ai exprimé tout naturellement l'Interfuit de la Photo par un parfait («cela a été »). L'aoriste est le temps de l'événement hors de la personne du narrateur : l'événement est le repère de la narration, non le narrateur (comme dans le parfait) ; et c'est bien ainsi que nous vivons une photo : comme l'image d'un événement pur, fiché dans l'Histoire sans aucune intervention humaine (le photographe, je n'y pense pas); dire que je sens la photo comme « objective », ce serait trop peu ; car l' « objectivité » est encore l'attribut d'un narrateur (même si cet attribut tend à l'absenter) : la photo est littéralement et absolument l'Histoire même : «ce qui a été », venu jusqu'à nous sans la médiation d'aucun témoin. (eneore une fois, phénoménologiquement, la photographie ne eompte pas). C'est là le paradoxe grammatical de la Photographie : d'être à l'aoriste, alors que quelqu'un l'a faite, mais ee quelqu'un est, selon ma conseienee, aussi abstrait, aussi absent qu'un dieu ${ }^{31}$.

Il faut tenir cette version manuscrite et les notes de cours dans les différences que les deux documents établissent : de l'un à l'autre, la réflexion s'est déplacée, et l'écriture du

31. R. Barthes, BnF, NAF 28630, La Chambre claire, manuscrit, fo 40. 
livre inverse l'hypothèse exposée à l'occasion de la séance du 15 février 1979 (le temps de la photo n'est pas le parfait, mais bien l'aoriste). Pour autant, le point à relever ne semble pas être dans cette infirmation - au moment de rédiger La Chambre claire - de l'intuition évoquée dans le cours. Et ceci pour deux raisons. D'abord parce que dans sa complémentarité à La Chambre claire, l'enseignement de 1979 permet à la pensée de s'exposer dans toute sa richesse, sans opération de sélection ou d'ordonnancement. C'est d'ailleurs bien ce qu'indiquait une des « chutes » du manuscrit de La Chambre claire: Barthes y appelait de ses vœux une «science des moires, des restes 32 ». Ensuite, parce qu'il paraît plus riche de s'interroger sur les motivations d'une telle suppression : à quelle décision s'articulet-elle ? Peut-être, en ce moment précis de la genèse, Barthes s'est-il, à l'exemple de Proust, trouvé divisé entre deux côtés - celui de l'Essai (développé au moyen de Benveniste) et celui du Roman (encore fantasmé). La séance du 15 février, consacrée à la photographie, aura été l'occasion de dévoiler les prémisses d'une analyse plus technique (quant à la valeur des temps, et quant à la validité d'une telle analogie) que la rédaction de l'ouvrage refuse, au profit du récit.

\section{Le côté du Roman}

C'est peu dire en effet que Barthes, dans le livre qu'il publie en 1980, propose un véritable récit de méthode, originé dans une anecdote aux allures de fable : «Un jour, il y a bien longtemps, je tombai sur une photographie du dernier frère de Napoléon, Jérôme (1852). Je me dis alors, avec un étonnement que depuis je n'ai jamais pu réduire : “Je vois les yeux qui ont vu l'empereur" » $(C C$, p. 791). À partir de cet événement fondateur, donnant son impulsion au trajet que suit l'ouvrage, l'auteur thématise ses errances, à la recherche d'un maître - en témoigne la question qui ouvre le deuxième chapitre : «Qui pouvait me guider ?». Cette question retrouve l'annonce jamais tout à fait explicitée au début de La Préparation du roman. Barthes évoque le cheminement de Dante : «(il y a un initiateur : Virgile - nous aurons le nôtre) » $(P R$, p. 26). Or, l'objet de cette quête initiée sous la bienveillance de la figure proustienne est double. Il porte tout d'abord sur la « spécialité » de la photo. L'impératif énoncé par Barthes lors du cours : « définir la spécificité de l'image photographique » (p. 114) - « spécificité » que ce dernier glose par <spécialité>, rappelant que les deux termes ont à l'origine le même sens - se retrouve ainsi sur le deuxième feuillet du manuscrit du livre, « Vouloir trouver à la Photographie sa spécialité33 ». Mais il porte également sur cette « tierce forme » dont la conférence désirait pénétrer le secret, dans l'intuition du repérage d'un bougé des frontières et assignations génériques au cœur de la pratique proustienne.

Barthes, ainsi, dévide le fil d'un récit qui est loin d'être linéaire. À l'occasion d'une parenthèse : « (je ne savais pas encore que de cet entêtement du Référent à être toujours là, allait surgir l'essence que je recherchais) » (CC, p. 793), se dessine le leurre d'un rendu au présent de la recherche. L'évidence s'impose - à savoir que l'auteur a remanié le texte en fonction de son point d'aboutissement et n'a pas simplement fait état, au fil de la plume, de ses errements et de ses impasses. «Que la logique diffère du chronologique », commente Bruno Clément, « c'est la règle en matière méthodique - règle absolue 34 ». Depuis la décision qui marque l'aventure de La Chambre claire (« Je décidai alors de prendre pour guide de ma nouvelle analyse l'attrait que j'éprouvais pour certaines photos », p. 803), jusqu'au dernier chapitre de la première partie, qui s'achève sur ces mots : «Je devais faire ma palinodie » (p. 836), l'organisation du livre lui permet de « se constituer en identité narrative $35 \gg$.

Du cours au livre, le trajet n'est en effet pas celui d'une exploration approfondie des quelques prémisses jetées rapidement à l'occasion d'une séance un peu hors propos ; il est bien plutôt celui d'une réduction progressive de la réflexion, à mesure que celle-ci trouve une forme à même de l'accueillir et que s'affermit son identité narrative. Que nous apprennent donc ces quelques pages des notes de cours consacrées à la photo ? Que tout est là, à disposition, et que les successives découvertes qui marquent le livre de 1980

32. Ibid., fo 41.

33. R. Barthes, NAF 28630, La Chambre claire, «Premier brouillon/ Chutes », fo 2 .

34. B. Clément, Le Récit de la méthode, Paris, Gallimard, 2005, p. 112. 35. Ibid., p. 22. 
ne sont pas liées à la genèse du texte : ce « récit, à la fois intellectuel et affectif », tel que Barthes le présente dans un premier prière d'insérer ${ }^{36}$, est d'abord quête d'une nouvelle « forme », de cette « tierce forme » : les dimensions intellectuelles et affectives soulignées par l'auteur rejoindraient le côté de l'Essai et celui du Roman, tels que repérés à l'œuvre (dans leur recroisement et leur dévoiement) chez Proust. Ce qu'indiquait déjà, si l'on accepte de s'y reporter, le feuillet 6 de la première version de la conférence : «Or ma pratique passée (déjà longue) est celle non de la Science (je n'y ai jamais cru) mais de l'Essai $\rightarrow$ Essai/Roman : c'est une bifurcation : cela fait deux côtés. Et voici qu'arrêté à ce croisement, je rencontre Proust ${ }^{37}$. » Ce croisement, cette bifurcation, annoncent une décision conquise de haute lutte, quant au côté à privilégier ; et si de cette lutte le dossier génétique de La Chambre claire ne porte pas explicitement la trace, la comparaison dynamique des différents documents (conférence, cours, brouillons du livre) permet, peutêtre, d'en ressaisir même imparfaitement les rouages. Dès lors, en confrontant les avant-textes du livre aux notes de cours, on peut envisager avec la juste distance nécessaire le protocole narratif mis en scène dans La Chambre claire. Il paraît possible ici de défendre une lecture à rebours du livre : ce dernier ne propose pas le trajet naïf d'une pensée qui évolue depuis l'étonnement saisissant Barthes devant la photo du dernier frère de Napoléon, jusqu'à la révélation d'une essence de la photographie qui serait advenue à mesure que s'écrivait l'ouvrage.

Bien au contraire, cette essence de la Photographie, Barthes, lors de la séance qu'il introduit, on s'en souvient, comme < digression>, la dévoile d'emblée : <mon hypothèse depuis longtemps formulée en moi, enfin formulée, esquissée, tentée, mais jamais explorée à fond, ce que je me propose de faire dans un travail prochain et j'espère proche : le noème de la photographie doit être cherché du côté de ce que j'appelle le cela a été, ça a été>38. S'il faut attendre le quarante-septième chapitre (le pénultième) du livre pour trouver cet aveu : « Le noème de la Photographie est simple, banal ; aucune profondeur : "Ça a été". Je connais nos critiques : quoi ! tout un livre (même bref) pour découvrir cela que je sais dès le premier coup d'œil ? » $(C C$, p. 880), c'est là un terme factice, en ce qu'il se présentait explicitement comme point de départ du développement du cours. Il faut entendre alors la protestation de l'auteur : "Oui, mais telle évidence peut être sœur de folie » (loc. cit.). Le livre permet ainsi à l'intuition (qui ne bougera pas, ne s'approfondira guère, n'était la découverte du punctum, approchée dans le cours via l'attention portée au détail) de se parer des atours de la démonstration. Et si La Chambre claire constitue de Barthes l'ouvrage le plus narratif, sa mise en récit ne serait-elle pas protection contre cette folie même ? Ce que le cours présentait comme « digression », le livre l'offre comme « progression », afin d'acclimater le sujet à cette évidence, de l'ordre de l'intraitable : en témoigne la mention qui ouvre le chapitre 32 de La Chambre claire : «Le nom du noème de la Photographie sera donc : "Ça-a-été", ou encore : l'Intraitable» (p. 851). Il s'agit donc de renverser le mouvement que l'on peut inférer, d'une première lecture, dans le livre : ce dernier détaillerait moins les étapes d'un cheminement ou d'une quête initiés lors du cours, et approfondis à cette occasion, qu'il n'exposerait les stades successifs d'une lucidité « intraitable » pourtant présente au moment de la « digression » sur la photo, durant la séance du 15 février 1979. Il s'agit dès lors moins d'observer comment, de La Préparation... à La Chambre claire, s'affermit ce que Barthes dit de la photo, que d'étudier comment l'auteur trouve à se protéger de la «blessure » qu'elle inflige au sujet 39 - et l'on rejoint là ce que la première version de la conférence dit de Proust écrivant la Recherche : «Poésie et Roman sont évidemment ici du même côté : ce qui peut guérir la Blessure 40 », en un pouvoir d'apaisement interdit à l'Essai.

36. BnF, NAF 28630, La Chambre claire, copie 1, « Note sur la photographie».

37. R. Barthes, BnF, NAF 28630, «Longtemps je me suis couché de bonne heure », première version manuscrite, f. 6 .

38. R. Barthes, séance du 15 février 1979 et $P R$, p. 114.

39. À l'opposé du studium, ce « champ d'étude », vague sinon «paresseux » qui nous attache à la plupart des photos, le punctum (cette relation d'élection à une photo) se noue pour Barthes autour d' " une essence (de blessure), ce qui ne peut se transformer, mais seulement se répéter sous les espèces de l'insistance (du regard insistant) » $(C C$, p. 828).

40. R. Barthes, «Longtemps je me suis couché de bonne heure », première version manuscrite, , f. 7. 


\section{Conclusion : un discours habitable}

Revenant sur l' « hésitation entre l'Essai et le Roman » qu'a sans doute affrontée Proust (et à laquelle, c'est là l'important, il a su apporter une réponse), Barthes explique, dans la première version manuscrite de la conférence «Longtemps... » :

Je retire de ceci, qui concerne Proust, les incitations personnelles suivantes (comme deux libertés créatrices) : 1) Ébranler n'est pas détruire : Proust n'a détruit ni le récit, ni le je, ni la biographie ; mais il les a déplacés : des formes du passé sont là, mais à une autre place : c'est la spirale (Peut-être une nouvelle conception, une révision du travail à faire - de l'avant-garde : ne pas emprisonner l'avant-garde dans ses tics) ; 2) Par les 3 points que je viens de dire : Proust a contaminé réciproquement l'Essai et le Roman : a lancé une tierce forme 41 (voir fig. 5).

Non pas détruire, mais déplacer, mais ébranler : tout le projet du dernier texte de Barthes semble ici résumé. La Chambre claire n'abandonne pas la dimension intellectuelle de son enquête sur la photographie (dimension exposée à l'occasion de la séance du 15 février 1979 de La Préparation du roman); mais elle ne lui sacrifie pas non plus l'intensité personnelle, la projection affective de sa démarche. Elle ne « détruit » pas les modalités énonciatives de l'essai ; elle en déplace les possibles. Ainsi ne comprendon vraiment le passage du cours au livre qu'au moyen des pistes lancées dans la conférence et de l'annonce d'une modification de l'écriture dans la définition (encore à venir) d'une « tierce forme ». L'hybridité de La Chambre claire - ni tout à fait Essai, ni tout à fait Roman ; à la fois Essai et Roman - relève de cette tierce forme, à laquelle il serait vain de donner une extension générique précise : dans le discours barthésien (celui du cours comme de la conférence), « tierce forme » et « roman » sont vite faits synonymes. Ils désignent tous deux cette « écriture médiate qui ne fait pas pression sur l'autre (le lecteur) $42 »$, dont l'« instance » serait « la vérité des affects, non celle des idées 43 ». Une forme qui exclurait moins qu'elle n'accueillerait, comme le réclament encore les premiers brouillons de la conférence :
Une nouvelle dialectique, une nouvelle topologie doit être cherchée : mettre le général (ou le Théorique, ou le Doctrinal) en position d'Indirect : donc : non pas l'abolir, le censurer (nous avons besoin du Théorique), mais le latéraliser, le prendre en écharpe. Pourquoi ? Parce qu'il nous faut un discours sans arrogance, sans terreur, sans répression : un discours habitable 44 (voir fig. 6).

Ce «discours habitable », l'écriture barthésienne en a inlassablement interrogé les contours, redéfini l'extension, et ce depuis Le Degré zéro de l'écriture (et la lutte qui s'y expose contre toute écriture empruntée du roman) au début des années cinquante, jusqu'aux Fragments d' un discours amoureux (et la défense de l' «intraitable 45 » d'une parole d'une grande solitude) en 1977 - date d'entrée de l'auteur au Collège de France. Tout le «moment Collège » (ces trois dernières années qui semblent d'abord consacrées à l'enseignement rue des Écoles ${ }^{46}$ ), si on le tient pour un moment pertinent, paraît tendre vers l'esquisse d'une nouvelle façon d'habiter la langue et ses contraintes (et ce afin de les déjouer). Ou plutôt : d'habiter l'hésitation, ce que, dans Le Neutre (et ce serait là une autre définition de cette « tierce forme » continûment désirée), Barthes avait désigné comme le souhait de « tenir l'intenable 47 », et qu'il a sans doute appris, repris certainement, de Proust.

\section{Ibid., f. 9}

42. Ibid., f. 6.

43. R. Barthes, « Longtemps... », art. cité, p. 469.

44. R. Barthes, BnF, NAF 28630, «Longtemps je me suis couché de bonne heure », première version manuscrite, f. 10.

45. On lit ce terme, d'une grande fortune dans l'imaginaire du « dernier Barthes » (je rappelle qu'il intervient dans la définition même du noème de la photographie ; voir $C C$, p. 851), dans la préface « Comment est fait ce livre » des Fragments (OC, t. V, p. 29).

46. Ce «moment » trouverait alors à s'unir autour d'une conception différente du geste d'écriture, d'un « écart entre la production écrite et la pratique de l'enseignement », rappelé par É. Marty : «si auparavant, lorsque Barthes animait un séminaire restreint à l'École pratique des hautes études, il a pu [...] être tenté de transformer tel ou tel cours en livre, cette hypothèse a totalement disparu » (« Avant-propos », dans R. Barthes, Comment vivre ensemble, op. cit., p. 9). Ainsi s'expliquerait la nécessité d'ouvrir l'étude de la genèse du dernier livre publié à une analyse plus large - ce que nous avons voulu proposer dans le cadre de cette réflexion.

47. R. Barthes, Le Neutre, éd. T. Clerc, Paris, Éditions du Seuil/IMEC, 2002, p. 39. 


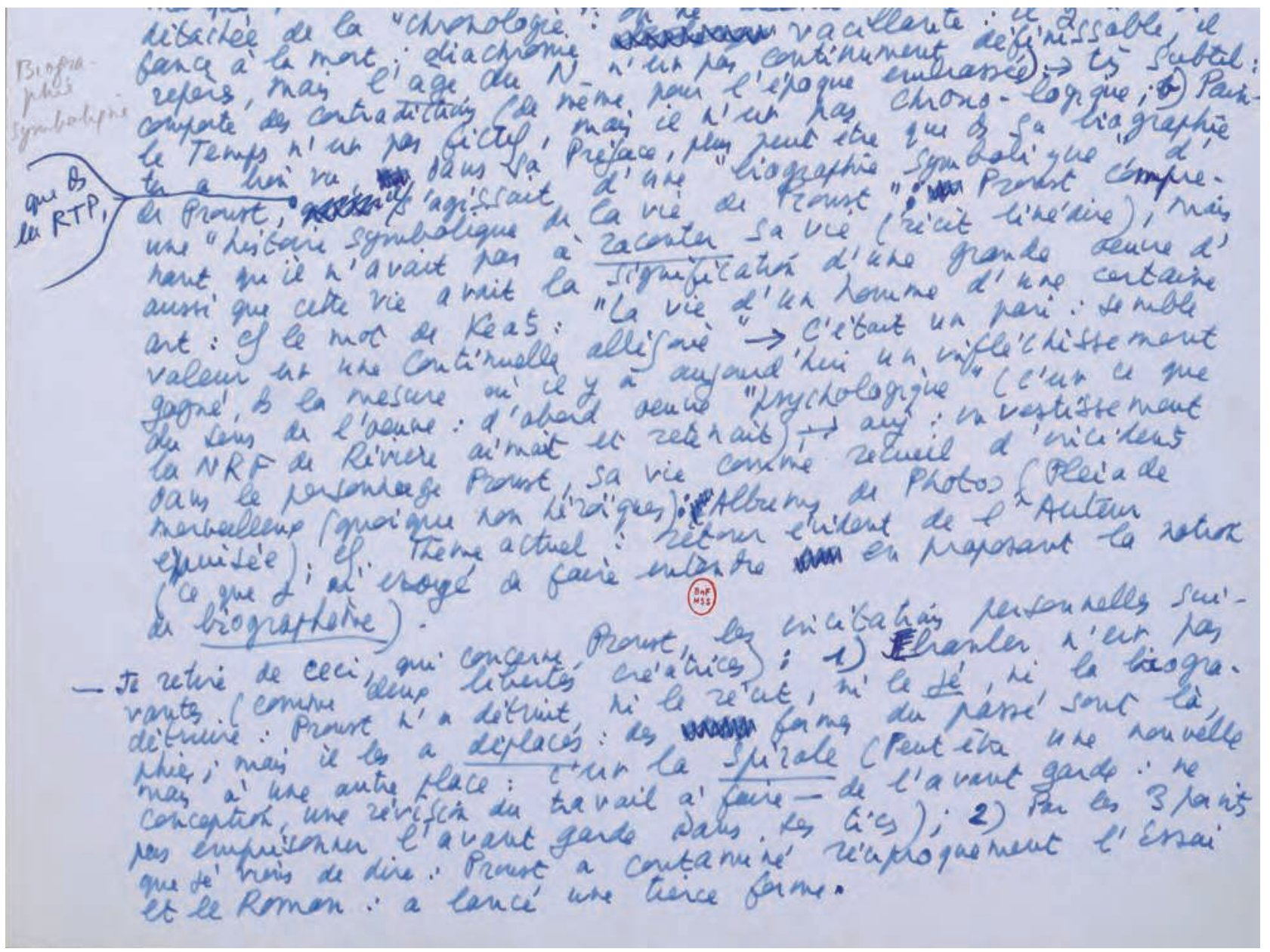

Fig. 5 : «Longtemps, je me suis couché de bonne heure », première version manuscrite de la conférence (BnF, NAF 28630, fo 9, détail)

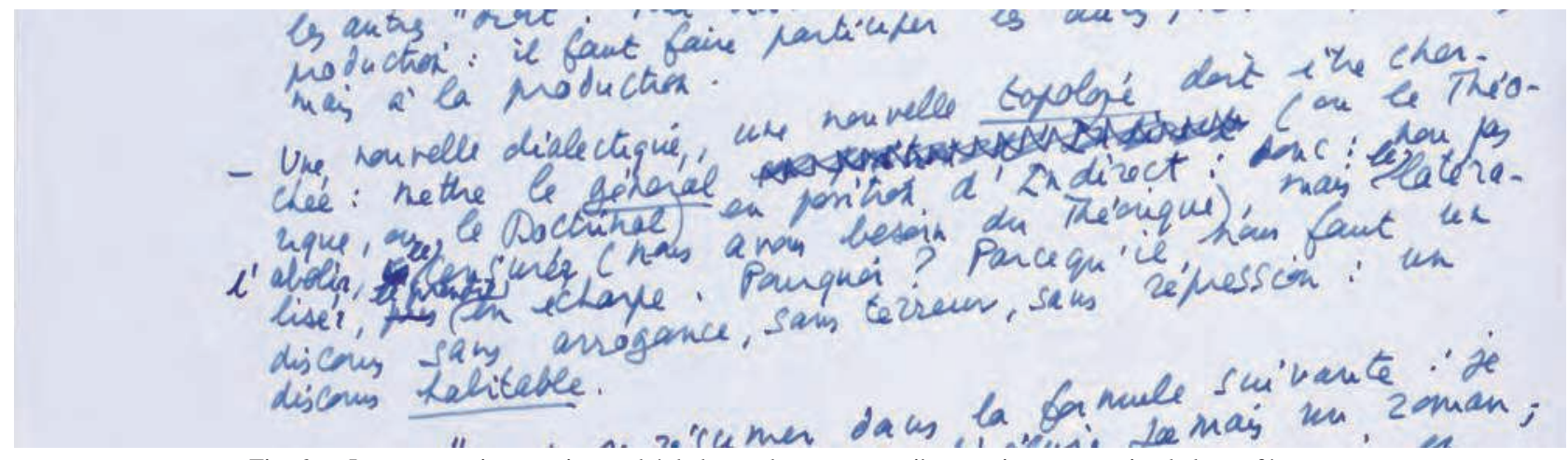

Fig. 6 : «Longtemps, je me suis couché de bonne heure », première version manuscrite de la conférence (BnF, NAF 28630, fo 10, détail) 
Guillaume Bellon est agrégé de lettres, docteur de 1'université Stendhal-Grenoble III, et titulaire d'un postdoctorat effectué à l'Université du Québec à Montréal. Il a dirigé pendant plusieurs années la revue en ligne de critique génétique Recto/Verso (<www. revuerectoverso.com>). Son livre, Une parole inquiète. Barthes et Foucault au Collège de France, est paru au printemps 2012 aux ELLUG.

guillaume.bellon@yahoo.fr

Résumés

\section{Barthes et l'" hésitation » proustienne, ou le cheminement des deux côtés de La Chambre claire}

Barthes, lisant Proust, insiste sur « 1'hésitation » qu'aurait affrontée l'auteur entre deux côtés : celui de l'essai et celui du roman. Or, La Chambre claire (dernier ouvrage du critique) semble témoigner d'une hésitation identique. La comparaison des manuscrits de cette «note sur la photographie » avec les réflexions livrées par Barthes à l'occasion d'une séance de La Préparation du roman, son cours au Collège de France, permet de nuancer l'idée d'une tentative de saisie intellectuelle de la photo. Il s'agirait en effet moins d'un texte de méthode (retraçant pas à pas les découvertes de l'auteur) que d'un récit - d'un roman.

When reading Proust, Barthes insisted on the "hesitation" that the writer would have had in choosing between two sides: the essay or the novel. Barthes' last work, La Chambre claire, seems to show an identical hesitation. Comparing the manuscripts of this "note on photography" with Barthes' reflections made during a presentation of La Préparation du roman, in his Collège de France class, enables us to qualify the idea of an attempt at an intellectual grasp of photography. This would be less the text of a method (retracing step by step the author's findings) than a story, a novel.

Barthes betont in seiner Proust-Lektüre dessen „Schwanken“ zwischen zwei Richtungen: zwischen Essay und Roman. Das letzte Werk des Kritikers, La Chambre claire, zeugt jedoch von einer ähnlichen Unentschlossenheit. Der Vergleich der Manuskripte dieser „Bemerkung zur Photographie“ mit den Überlegungen, die Barthes anlässlich einer Sitzung seines Kurses La Préparation du roman am Collège de France vorgetragen hat, erlaubt den Versuch einer intellektuellen Erfassung des Photos. Es würde sich dabei effektiv weniger um einen methodischen (Schritt für Schritt die Entdeckungen des Autors darstellenden) Text handeln, sondern um eine Erzählung - einen Roman.
Barthes, leyendo a Proust, insiste acerca de la "indecisión" a la que se vio confrontado el autor entre dos polos: el del ensayo y la novela. Ahora bien, La cámara lúcida (último libro del crítico) parece poner de manifiesto una indecisión similar. La comparación de los manuscritos de esta "nota sobre la fotografía" con las reflexiones enunciadas por Barthes en una sesión de su curso en el Colegio de Francia, titulado La preparación de la novela, permite matizar la idea de una tentativa de comprensión intelectual de la fotografía; se trataría, en efecto, más que de un texto de método (que restituye paso a paso los descubrimientos del autor) de un relato, de una novela.

Barthes, leggendo Proust, insiste sull'"hésitation" che avrebbe affrontato l'autore nei due piani di scrittura: quello del saggio e quello del romanzo. Tuttavia, ne La Chambre claire (ultimo studio del critico) egli sembra mostrare una identica "hésitation". Il confronto della lezione dei manoscritti che tramandano questa "note sur la photographie" con le riflessioni dettate da Barthes nel corso di una seduta de La Préparation du roman, il suo corso al Collège de France, permette di attenuare l'idea di un tentativo di comprensione intellettuale della foto. Sembra infatti che si tratti meno di un testo di metodo (traccia passo per passo le conclusioni dell'autore) che di un racconto - di un romanzo.

Barthes, lendo Proust, chama a atenção para a "hesitação" que o teria dividido entre dois lados: o ensaio e o romance. Ora, $L a$ Chambre claire (último livro do crítico) parece testemunhar uma hesitação idêntica. A comparação entre os manuscritos dessa "nota sobre a fotografia" e as reflexões expostas numa sessão de La Préparation du roman, curso de Barthes no Collège de France, permite matizar a ideia de uma promoção intelectual da fotografia. Seria questão menos de um texto sobre o método (rastreando passo a passo as descobertas do autor) e mais de uma narrativa - de um romance. 